\title{
A NOTE ON $k$-CRITICALLY $n$-CONNECTED GRAPHS ${ }^{1}$
}

\author{
R. C. ENTRINGER AND PETER J. SLATER
}

\begin{abstract}
A graph $G$ is said to be $\left(n^{*}, k\right)$-connected if it has connectivity $n$ and every set of $k$ vertices is contained in an $n$-cutset. It is shown that an $\left(n^{*}, k\right)$-connected graph $G$ contains an $n$-cutset $C$ such that $G-C$ has a component with at most $n /(k+1)$ vertices, thereby generalizing a result of Chartrand, Kaugars and Lick. It is conjectured, however, that $n /(k+1)$ can be replaced with $n / 2 k$ and this is shown to be best possible.
\end{abstract}

The terminology and notation of [1] will be used throughout. In [4] the notion of a critically connected graph was generalized as follows. A graph with connectivity $n$ is $k$-critical if whenever $S$ is a vertex set with $|S| \leqslant k$ the connectivity of $G-S$ is $n-|S|$. That is, every vertex set with no more than $k$ members is contained in an $n$-cutset or a trivializing set of $n$ vertices. Such a graph will be said to be $\left(n^{*}, k\right)$-connected.

Chartrand, Kaugars, and Lick have shown [2] that an $\left(n^{*}, 1\right)$-connected graph, $n \geqslant 2$, contains a vertex of degree at most $3 n / 2-1$. This would follow, of course, if it were known that an $\left(n^{*}, 1\right)$-connected graph $G$ contained an $n$-cutset $C$ such that $G-C$ contained a component with at most $n / 2$ vertices. Our object in this note is the generalization of the latter statement. To this end we develop the following notation.

Given a graph $G$ with connectivity $n$ let $C_{G}$ be the family of all $n$-cutsets of $G$. If $C$ is a member of $C_{G}$ denote by $\nu(C)$ the number of vertices in a smallest component of $G-C$. Finally, let $r(G)=\min \nu(C)$ where the minimum is taken over all members $C$ of $C_{G}$. That is, $r(G)$ is the order of a smallest component that can be obtained by removal of an $n$-cutset from $G$.

THEOREM. If $G$ is an $\left(n^{*}, k\right)$-connected graph with $1 \leqslant k<n$ then $r(G) \leqslant$ $n /(k+1)$.

Proof. Choose an $n$-cutset $C$ of $G$ so that $\nu(C)=r \equiv r(G)$, and let $R$ be the vertex set of a component of $G-C$ chosen so that $|R|=r$. Our proof will use the following property of $R$ :

(i) An $n$-cutset $D$ of $G$ that contains a vertex of $R$ contains all vertices of $R$. To show this we let $L$ be the vertex set of $G-C-R$, let $T$ be the vertex set

Presented to the Society, January 28, 1977 under the title $k$-critically $n$-connected graphs; received by the editors February 15, 1977.

AMS (MOS) subject classifications (1970). Primary $05 C 99$.

Key words and phrases. $n$-connected, critical, graph, component.

${ }^{1}$ This work was supported by the U. S. Energy Research and Development Administration (ERDA) Contract No. AT(29-1)-789.

○ American Mathematical Society 1977 
of one component of $G-D$ and let $B$ be the vertex set of the remaining components of $G-D$ (see Figure 1).

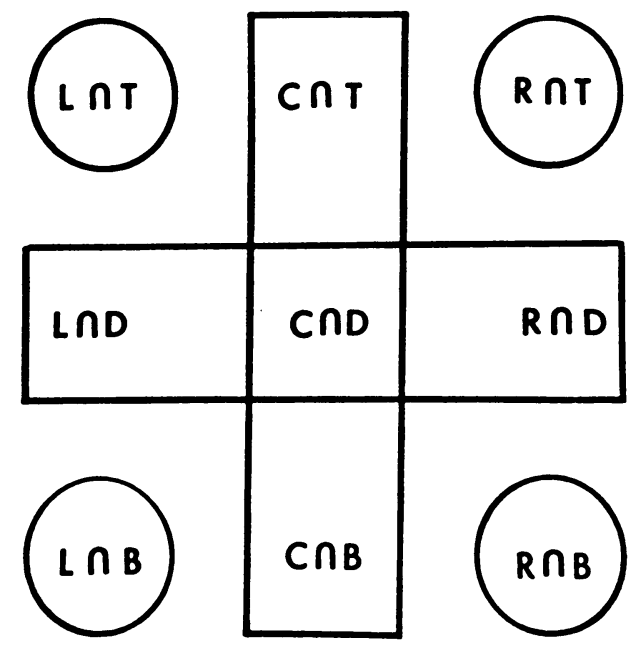

FiguRE 1

Since it is easy to argue that if $L \cap T$ is not empty then $(L \cap D) \cup(C \cap$ $D) \cup(C \cap T)$ is a cutset of $G$, we suppress the details and note that analogous results hold for $L \cap B, R \cap T$, and $R \cap B$.

We can now conclude that at least one of the sets $L \cap T$ and $R \cap B$ is empty. If this were not so we must have $|(L \cap D) \cup(C \cap D) \cup(C \cap T)| \geqslant$ $n$ and $|(R \cap D) \cup(C \cap D) \cup(C \cap B)| \geqslant n$. But equality must hold for both expressions since $|C|=|D|=n$. This is impossible, however, since it implies $r \leqslant|R \cap B|<|R|$. By similar argument one of the sets $L \cap B$ and $R \cap T$ must be empty.

Now, if (i) is not true, we may assume $R \cap T \neq \varnothing$ so that $L \cap B=\varnothing$. If, also, $R \cap B \neq \varnothing$ then $L \cap T=\varnothing$ so that $|R \cap D|<|R| \leqslant|L|=|L \cap D|$. Consequently, $|R \cap D|<\frac{1}{2}(n-|C \cap D|)$, which, in turn, gives

$$
2 n \leqslant|C \cap T|+2|C \cap D|+2|R \cap D|+|C \cap B|<|C|+n \text {. }
$$

Since this is impossible we must have $R \cap B=\varnothing$ so that $|C \cap B|=|B| \geqslant$ $|R|>|R \cap D|$. But this implies $(C \cap T) \cup(C \cap D) \cup(R \cap D)$ is a cutset with fewer than $n$ vertices. Consequently (i) is proven and we now show:

(ii) The theorem holds for $k=1$.

We assume otherwise and, referring to Figure 1 , note that $|R \cap D|=|R|$ $>n / 2$ implies $|L \cap D| \leqslant|(L \cap D) \cup(C \cap D)|<n / 2$, so that we assume $L \cap T \neq \varnothing$ and, consequently, must have $|C \cap T|>n / 2$. This implies $|C \cap B|<n / 2$ so that $L \cap B$ cannot be empty. But then $(L \cap D) \cup(C \cap$ D) $\cup(C \cap B)$ is a cutset with fewer than $n$ vertices, which cannot be. Hence (ii) holds and we now show:

(iii) $G-R$ is an $\left((n-r)^{*}, k-1\right)$-connected graph. 
$G-R$ is obviously $(n-r)$-connected. To show that any $k-1$ vertices of $G-R$ lie in an $(n-r)$-cutset of $G-R$ we choose any such $(k-1)$ set $S$ of $G-R$ together with one vertex $p$ of $R$ and extend this to an $n$-cutset $S^{\prime}$ of $G$. By (i) $R$ is a subset of $S^{\prime}$ so that $S^{\prime}-R$ is an $(n-r)$-cutset of $G-R$ containing $S$, and (iii) is proven.

We can now complete the proof of the theorem by induction on $k$. We may assume it holds for all $(k-1)$-critically connected graphs with $k>1$. Then, by (iii), $G-R$ contains an $(n-r)$-cutset $C^{\prime}$ such that $G-R-C^{\prime}$ has a component $R^{\prime}$ with at most $(n-r) / k$ vertices. But since $R \cup C^{\prime}$ is an $n$-cutset of $G$ we must have $r \leqslant(n-r) / k$, i.e., $r \leqslant n /(k+1)$, and the proof is complete.

We obtain the following consequence immediately upon consideration of the degree of a vertex in the set $R$ described in the proof of the theorem.

CoRollary. An $\left(n^{*}, k\right)$-connected graph contains a vertex of degree at most $(k+2) n /(k+1)-1$.

In particular, we can conclude that any $\left(n^{*}, k\right)$-connected graph with $k>(n-1) / 2$ has a vertex of degree $n$. In the cases $n=2$ and 3 more is known, however. L. Nebesky [5] has shown that a $(2,1)$-connected graph with at least six vertices contains four vertices of degree 2 and this result is best. The authors [3] have shown that a $(3,1)$-connected graph contains at least two vertices of degree 3 and this result is best. Suppose, now, that $G$ is a $(4,2)$-connected graph. Then $G$ has a vertex $p$ of degree 4 so that, by (iii) above, $G-p$ is a $(3,1)$-connected graph and consequently has two vertices of degree 3. $G$, then, had at least three vertices of degree 4 . The possibility that such properties are not restricted to graphs with low connectivity can be made explicit as follows.

CONJECTURE 1. An $(n, k)$-connected graph with $k \geqslant(n-1) / 2$ contains at least two vertices of degree $n$.

We do not believe that the result of the theorem is best except at $k=1$ and $k=[n / 2]$, but, however, do have some confidence in the following conjecture.

CONJECTURE 2. If $G$ is an $\left(n^{*}, k\right)$-connected graph then $r(G) \leqslant n / 2 k$.

We will describe a class of graphs, Figure 2, showing that this conjecture, if true, is best possible. For each $k \geqslant 1$ and $r \geqslant 1$ we define a graph $G_{k, r}$ as follows. The vertex set of $G_{k, r}$ consists of $2 k+2$ sets $S_{1}, \ldots, S_{2 k+2}$ of $r$ vertices each. Two vertices are adjacent if and only if they lie in sets $S_{i}$ and $S_{j}$ such that $i-j \not k+1 \bmod (2 k+2)$. It is obvious that $G_{k, r}$ has connectivity $2 k r$ and that $r\left(G_{k, r}\right)=r$. Also, for any choice of a set $S$ of $k$ vertices of $G_{k, r}$ there will be an $i$ such that neither $S_{i}$ or $S_{i+k+1}$ (indices reduced $\bmod (2 k+$ 1)) contains a vertex of $S$. Consequently, $S$ can be completed to a $2 k r$-cutset and so $G_{k, r}$ is a $\left((2 k r)^{*}, k\right)$-connected graph.

Conjecture 2, in addition to being correct, with proper interpretation, for noncritical graphs, i.e. at $k=0$, would imply that if $G$ is an $(n, k)$-connected 


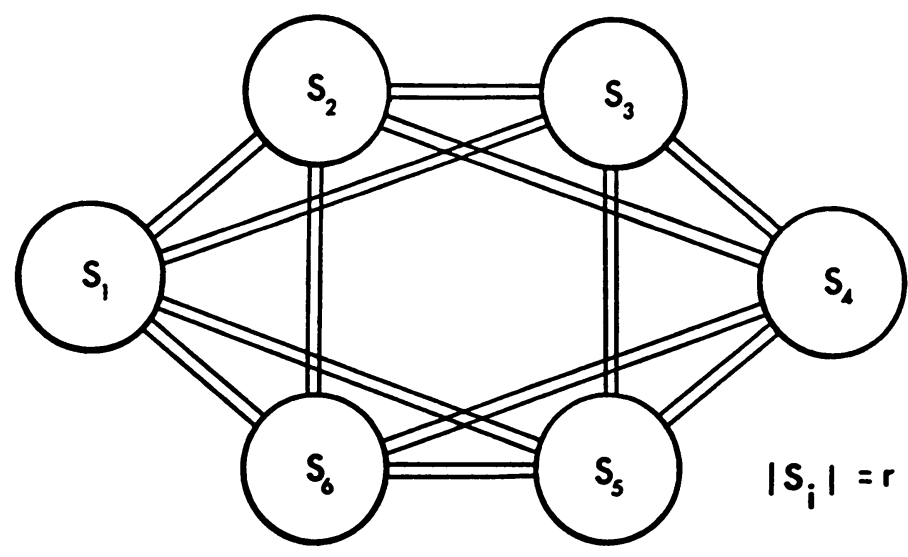

FIGURE 2

graph then either $k<[n / 2]$ or $k=n$ and $G=k_{n+1}$. This latter implication has been conjectured by Slater [4].

NOTE ADDED IN PROOF. W. Mader has kindly informed us that property (i) in the proof of the Theorem had been previously proven by him [Eine eigenschaft der atome endlicher graphen, Arch. Math. (Basel) 22 (1971), 333-336.]

\section{REFERENCES}

1. M. Behzad and G. Chartrand, Introduction to the theory of graphs, Allyn and Bacon., Boston, 1971.

2. G. Chartrand, A. Kaugars and D. R. Lick, Critically n-connected graphs, Proc. Amer. Math. Soc. 32 (1972), 63-68.

3. R. C. Entringer and P. J. Slater, $A$ theorem on critically 3-connected graphs, Nanta Math. (to appear).

4. Stephen Maurer and Peter J. Slater, On n-connected and $k$-critical graphs, Discrete Math. (to appear).

5. L. Nebesky, On induced subgraphs of a block, J. Graph theory 1 (1977), 69-74.

Department of Mathematics, University of New Mexico, Albuquerque, New Mexico 87131

Sandia laboratories, Albuquerque, New Mexico 87115 